\title{
El Ebro y Zaragoza
}

\section{Jordi Sardà Ferran ${ }^{1}$ Indibil Solans Ibañéz ${ }^{2}$, Mikel Burgui Moreno ${ }^{3}$}

Centre de Recerca Urbana del Camp (CRUC). Unidad Predepartamental de Arquitectura. Escuela Técnica Superior de Arquitectura. Universitat Rovira i Virgili. Reus, Tarragona, España E-mail: 1sardaprim15@gmail.com, ${ }^{2}$ solanssi@gmail.com, ${ }^{3}$ burgui.mikel@gmail.com

\begin{abstract}
Resumen. Todas las ciudades establecen una relación saprofita con su rio que primero es vía de navegación y defensa, después un vado posible, que la construcción del primer puente - de piedra- confirma como nexo entre riberas y obligado cruce de caminos. El río es garantía de agua limpia y alcantarilla, de huerta feraz y de energía. Solo algunas veces el agua toma la palabra y pretende recuperar derechos primigenios. Pero la ciudad siempre gana. El caso de Zaragoza y el Ebro es un perfecto paradigma.

Esta comunicación parte de un programa de estudio y un taller de proyectos sobre toda la Cuenca del Ebro, entendida como un territorio, donde las condiciones de la historia han establecido gran diversidad de fueros y lenguas generando una cultura riquísima. Los cursos y el taller, a la manera del viaje por el Danubio de Claudio Magris, reflexionan sobre las distintas vocaciones de las tierras y del agua. Pero siempre siendo conscientes de la centralidad de Zaragoza y de su mesura o desmesura urbana; en fin de la condición de la ciudad como dueña absoluta del río. Los regadios, canales y huertas, las centrales nucleares o térmicas, la industria y población acumuladas, parecen confirmar en toda la Cuenca que todo trabaja para Zaragoza, incluso el Ebro. Compararemos los sistemas de ciudades de Aragón con los de la Cuenca entera y comprobaremos que la especial posición en el centro del inmenso llano, superando la presencia del pequeño Huerva, ha permitido a Zaragoza crecer sin límites, incorporando finalmente "a su cuerpo" el propio Ebro. Una mirada minuciosa a la cartografía histórica ha permitido confirmar que, a través de encuentros y desencuentros, la ciudad siempre salda a su favor. Murallas, templos, ferrocarril, estación, eventos, cambios de escala, todo tiene que ver con la relación saprofita con el río. Efectivamente el río es la ciudad, y viceversa
\end{abstract}

\section{Palabras clave: Cuenca del Ebro, Valle del Ebro, Zaragoza,} sistemas de ciudades, relación saprofita ciudad-río

\section{Contexto}

Esta comunicación se inscribe en el marco de un programa académico de la Escuela Técnica Superior de Arquitectura de Reus URV que propone el estudio de la Cuenca del Ebro en tres cuatrimestres consecutivos a lo largo de tres cursos. En la Escuela de Reus la docencia de urbanismo y de proyectos es compartida, por este motivo parece adecuado establecer marcos territoriales amplios donde lo urbano y lo arquitectónico se encajan y relacionan íntimamente con la geografía y el paisaje.

Con esta voluntad el curso- tercero de un programa académico de cinco- ha planteado ejercicios de lectura, dibujo e interpretación a escala 1/100.000 que han llevado toda "la cuenca del Ebro" al aula. Luego una sucesión de múltiples "Biopsias territoriales" realizadas a escala 1/10.000 han permitido visualizar -a través de plano-ventana de 120x30 cm.- "Las formas del territorio fluvial", que enmarcan las ciudades dialogando estratégicamente con sus territorios irrigados y feraces. 
Entre las ciudades escogimos dos: Tortosa ${ }^{1} \mathrm{y}$ Tudela ${ }^{2}$ similares por sus medida, historia y transcendencia urbana. Ambas poseen puentes de piedra, castillo, murallas, catedral y obispado $\mathrm{y}$ en consecuencia, ambas son cabecera de un territorio transformado por el río y sus canales, que han sido determinantes para la fortuna de la ciudad. De ambas hemos descubierto y dibujado sus Formas de Crecimiento Urbano 3. El curso acaba con dos pequeñas, pero certeras propuestas de transformación y de desarrollo urbano. La primera en Tudela y en Tortosa proponiendo un nuevo barrio, en algunos núcleos de menor intensidad Deltebre ${ }^{4}$, Mequinessa ${ }^{5}$ Miravet ${ }^{6}$ y Valtierra ${ }^{7}$ núcleos situados todos ellos en los territorios fertilizados por el agua del Ebro. Imaginamos intensificaciones de tejidos urbanos existentes, con arquitecturas y técnicas contemporáneas pero coherentes con las formas y usos preexistentes

Hemos viajado a los territorios y ciudades estudiados- Tudela, Tortosa, Zaragoza- y a la vez leído "Danubio " de Claudio Magris y "Cami de Sirga " " de Jesús Moncada. Y, con las imágenes de las ruinas iberas - "Castellet de Banyoles 10"- las centrales nucleares - Ascó ${ }^{11}$ o los canales - Tauste, LodosaImperial ${ }^{12}-$, construido un primer relato sobre las propiedades de la Cuenca, abocada a la producción de energía para usos ajenos al territorio y a la agricultura intensiva, un territorio punteado por decenas de ciudades de todo tamaño y posición, donde Zaragoza reina sobre todas las demás. Territorio difícil de leer y explicar en su complejidad y variedad.

\section{Objetivos}

La Cuenca del Ebro, como casi hemos dicho, es un enorme territorio de $85.000 \mathrm{~km}^{2}$ - mayor que muchos países europeos, mayor que Austria o Serbia-. Se extiende por diferentes: estados, reinos, principados, comunidades autónomas y provincias. Territorio que por su gran extensión presenta notables diferencias climáticas y paisajistas: desde un Pirineo lluvioso, a los desiertos de la Bárdenas Reales ${ }^{13}$ y a un mediterráneo deltaico. Administrativamente, contiene 1.700 municipios y está gestionado, -respecto al agua-, por diferentes instituciones;
- Comunidades de Regantes, Compañías Eléctricas, Agencias del Agua o de residuos ... todas, por fortuna, bajo la tutela de la Confederación Hidrográfica del Ebro $^{14}$, con sede en Zaragoza. Gracias o por culpa de los embalses construidos en su curso, apenas es navegable. Tampoco se puede reseguir por carretera o ferrocarril. Solo desde el aire se pueden percibir con claridad sus propiedades y sus límites. El río y su Cuenca -el territorio que baña y fertiliza-, es un lugar repleto de fronteras y barreras, que constituye, solo sobre el papel, un territorio compartido. ¿Es la Cuenca un concepto abstracto o existe realmente? ¿Hay alguna relación de parentesco entre Frías y Flíx, Miranda y Tortosa? ¿Hay alguna calle, plaza, edificación o ventana en Sástago similar a las de Ascó o Deltebre?. Este es el objetivo del curso y del articulo: preguntarnos sobre las condiciones urbanas compartidas en el ámbito de la Cuenca del Ebro. También y, no es tema menor, esta es una propuesta académica donde la investigación y docencia quieren ir de la mano y al unisonó. Esta comunicación es prueba de ello.

\section{Metodología}

A partir del curso propuesto y expuesto $\mathrm{y}$ en paralelo, hemos planteado una mirada intensa y propia a Zaragoza. En la visita a la Confederación Hidrográfica del Ebro los estudiantes explicitaron "queremos trabajar en Zaragoza. Esa es una buena ciudad". Zaragoza será tema de estudio y propuesta en el último año de nuestro programa de curso-trilogía. Proponemos ahora un primer acercamiento a la ciudad, a su territorio y al río desde varios puntos de vista, todos elaborados a partir de los trabajos producidos en el curso por sus estudiantes y profesores invitados. Hemos agrupado la reflexión bajo 4 títulos:
a) La demografía
b) El peso y la forma de las ciudades
c) El ferrocarril como elemento vertebrador del territorio.

d) Las cartografías de Zaragoza.

\section{La demográfica.}

A partir de los datos de población del INE y 
otros organismos oficiales hemos elaborado una gráfica de la evolución demográfica de Zaragoza comparada con la ciudades medias y mínimas de toda la Cuenca y especialmente de Aragón, que nos permitirán una reflexión demográfica. ¿Por qué Zaragoza ha crecido tanto y otras ciudades de la Cuenca del Ebro tan poco o incluso decrecido? ¿Por qué el Valle del Ebro estuvo tejidos por un sistema urbano de gran densidad y potencialidad y ahora la población -que ha crecido notablemente en su conjunto- se ha concentrado solo en las grandes ciudades $y$ en Zaragoza especialmente? -Zaragoza tiene hoy la mitad de la población de Aragón cuando en el censo de Madóz 1857 solo poseía una décima parte- y ninguna ciudad de Aragón ni del Valle ni de la Cuenca del Ebro ha acumulado población y riqueza con tanto ímpetu y fortuna.

Tortosa otra ciudad estratégica, situada en la puerta fluvial del Delta y en el paso obligado del río -entre Valencia y Barcelona-, contaba con 25.000 habs. en el censo de 1857, la mitad de Zaragoza, pero hoy (2016) Zaragoza supera los 700.000 habs. y Tortosa (2016) no llega a los 35.000 habs. Tortosa, tiene río, agua, tierra fértil e incluso mejor clima que Zaragoza y ambas comparten posición estratégica. ¿Por qué Zaragoza ha ido sumando municipios a su territorio mientras Tortosa los perdía y reducía así su capacidad táctica y técnica al tiempo que su demografía? El crecimiento desmesurado de Zaragoza y el despoblamiento de Aragón son vasos comunicantes, es un fenómeno tan estudiado como injustificado, según nuestro parecer y el de Mario Gaviria. Vamos a avanzar algunas líneas de estudio.

La capacidad del lugar.

La capitalidad.

La industrialización.

La capacidad del lugar. El término municipal de Zaragoza es hoy el mayor de Aragón (974 $\mathrm{km})$. y limita con 29 términos municipales, todos claro está, de menor extensión. Este fenómeno de absorción explicita del propio contexto tiene como contra partida la inexistencia de ciudades periféricas a Zaragoza, ciudades que, a la larga, construirían su área metropolitana. En este sentido observamos que otras ciudades menos ambiciosas o menos capaces, Huesca, por ejemplo, han compartido su urbanidad con un interesante sistema de ciudades mínimas: Barbastro, Binefar, Fraga; Monzon. Incluso Logroño -otra ciudad de ribera- ha conseguido un crecimiento fulminante sin cuestionar la existencia de un riquísimo sistema urbano a lo largo de la rivera del rio Ebro, Alfaro, Calahorra, Cintruenigo, Corella, Haro.

La capitalidad. La ciudad ha acumulado el gobierno del reino, el de todas las instituciones, las provinciales, las económicas y las instituciones más notables -Universidad, Confederación Hidrográfica del Ebro-. Superada la devastación demográfica producida por los franceses en 1808 -cuando Zaragoza perdió dos tercios de su población- la ciudad ha crecido en habitantes de manera continuada. La guerra civil española 1936-1939 supuso "al contrario" un estímulo al crecimiento, dado que Zaragoza permaneció siempre en el bando nacional y fuera del frente -impresiona ver Aragón partido verticalmente durante tres años por la línea del frente de combate- ${ }^{15}{ }^{16} \mathrm{La}$ ciudad de Teruel no tuvo la misma fortuna. Quizá, en consecuencia, la provincia de Teruel es la única de Aragón y de las pocas de España que tiene un crecimiento vegetativo negativo y no para de perder población. ${ }^{17} 18$

La industrialización. Además, el hecho de haber acertado de bando, confirmó a Zaragoza como ciudad segura y favorecida luego por el nuevo régimen. Ahí radicaría el tercer vector de su éxito, la industrialización de la ciudad. En efecto, las industrias agrícolas iniciales dejaron paso a una gran variedad de actividades productivas poco especializadas que han convertido a Zaragoza en una verdadera ciudad industrial, compitiendo con Barcelona o Bilbao.

La ciudad ha aprovechado bien los recursos: el agua, la energía, el suelo y el propio Ebro, que ella considera suyo. ¿Cómo debe de relacionarse la capital con su entorno? Y ¿Cómo debe de gobernarlo? ¿Cómo debe afrontar la nueva industrialización? ¿Debe especializarse, como ha hecho Toulouse? ¿Debe Zaragoza seguir creciendo aprovechando la falta de competencia de otras ciudades o debe reconsiderar que la vitalidad demográfica no implica siempre mejor calidad de vida? 


\section{El peso y la forma de las ciudades}

Es una comparación de la masa urbana de varias poblaciones mínimas y medianas con Zaragoza. Es un dato físico referido a la medida y escala urbanas y a menudo distinto al dato escueto de la población. Es una reflexión dibujada y complementaria a la reflexión demográfica. Nos permite comprobar como la resiliencia de lo urbano convive con todas las fases del crecimiento físico de las ciudades, independientemente de sus medidas.

En el pasado eran notorias las barreras físicas al crecimiento: por hechos naturales, ríos, montes, mar o por las líneas de defensa construidas por los hombres -murallas, siempre pendientes de ser demolidas o superadas-. Hechos que explicaban y justificaban la forma urbana de las ciudades. Hoy, la necesidad de suelos para usos industriales o logísticos sin restricciones de medidas, abiertos a las nuevas movilidades, y las nuevas movilidades han supuesto continuos crecimientos de la masa urbana sin apenas crecimientos demográficos.

¿Por qué Tarazona -que apenas crece demográficamente- lo hace en superficie urbana? Seguramente, por los mismos motivos que Alcañiz o Jaca que si crecen en habitantes. Claramente, el uso no restrictivo del suelo es el responsable. Es decir, crece la masa, pero no el peso y así es en todas las ciudades. Parece que Teruel especialmente, pero también Alcañiz, Tarazona e incluso Jaca han debido vincular su crecimiento a su topografía y geografía. Huesca ha sido más libre y su crecimiento ha sido más pautado. Pero Zaragoza ha sido lógicamente la ciudad que más ha crecido, con mayor libertad y capacidad de superar los impedimentos al crecimiento urbano. ¿Son sus abundantes vías y el ferrocarril, los responsables? ¿Qué papel ha jugado el planeamiento reglado? ¿Ha sido este el motor intermediario a la extensión urbana?

Está clara la vocación de la ciudad de crecer y de hacerlo en todas direcciones a lo largo de todas las vías, pero usando el río casi como centro de gravedad. En este sentido es curioso que haya sido para Zaragoza más fácil superar el Huerva -cubriéndolo y urbanizándolo- o el propio Ebro -reiteradamente puenteado- que el Gallego, con el cuál la ciudad tiene una relación más dubitativa, usando a favor y en contra su inundabilidad de su territorio.

Entendemos que esta es la vía oportuna para repensar los sistemas urbanos, que no pueden -ni a través del planeamiento, ni de la definición exacta de la vocación de los territorios-, establecer límites fijos entre lo urbano y lo no urbano. ${ }^{19}$

Así, algunos espacios industriales de Zaragoza deberán devenir -muy prontobarrios residenciales y algunos huertos urbanos de Tarazona o Jaca constituir patrimonio paisajistico y tejido urbano. Los espacios vacíos son también en las ciudades del Ebro tan importantes como los de urbanidad definida. Un concepto, este, que no es nuevo pero ahora es universalmente reconocido.

Pero volvamos a la reflexión sobre la demografía, el peso y la forma para confrontar la idea del crecimiento a la del "cascaron vacío".

Quizá deberemos valorar la historia, "las vidas vividas y acumuladas en cada lugar", y no solo el crecimiento demográfico o el extensivo. Así daremos el mismo valor a las vidas de los habitantes actuales, y a las de los que han sido. Quizá Albalate, Daroca o Tarazona dejarán de tener una línea demográfica descendente, siempre acumularán vida. Quizá asi podrán competir con mayor propiedad y orgullo con Zaragoza, que siempre tiene las de ganar. Sera una buena manera -a nuestro entenderde establecer una comparación en positivo, acumulando vidas y tiempos -peso- a una forma donde llenos y vacíos también suman y tienen idéntico valor.

\section{El ferrocarril como elemento vertebrador del territorio.}

El mapa de: Explotación, construcción y proyecto de los ferrocarriles de España y Portugal de Alfredo Forcano Catalán de $1949^{20}$ es una pieza rara pues recoge en una sola cartografía: pasado, presente y futuro. Es, a la vez, deseo y realidad producido en un tiempo (1949) en que el tren -aún no estaba amenazado por el automóvil- era la única esperanza de modernización de la movilidad en España. No es fácil - ni con la ayuda de los colores distinguir la red existente de la proyectada y 
de la en ejecución de las solo imaginadas. $\mathrm{Ni}$ las de transportes de viajeros, ni de las mineras e industriales. Están todas superpuestas, pero juntas, dibujan un sistema, una trama que teje todo el territorio del mapa. Sobre todo, en el Valle del Ebro y especialmente, en Aragón. Aparecen y se hacen notar los nodos, cruces y bifurcaciones - Zaragoza es el más claro, pero también lo son Miranda de Ebro, Pamplona, Castejón y Lleida, estos aun lo son hoy, pero otros: Soria, Cariñena o Teruel han perdido esta condición por completo.

Ninguno de los ferrocarriles proyectados se ha acabado construyendo. Otros, en obras, no se han terminado e incluso algunos se han desmantelado. Las conexiones ferroviarias de Alcañiz con Lleida, o Tortosa, o de Teruel con Soria nunca han cuajado. La red se ha convertido en madeja, para el uso de un privilegiado Ave "solo para viajeros" mientras el territorio vacío no dispone de transporte ferroviario. El coche ha ganado. ${ }^{2122}$

Hoy solo cuatro trenes cubren el trayecto Zaragoza-Caspe y viceversa, tardan 1 h $21 \mathrm{~min}$ (ida-vuelta) para cubrir $81 \mathrm{Km}$, pero hay 25 trenes para ir y volver de Zaragoza a Lleida y la mayoría del trayecto de $(128 \mathrm{Km})$ se puede hacer en sólo 43 minutos. Con Teruel pasa otro tanto, cuatro trenes diarios a Zaragoza y un tiempo medio de 2 horas y 21 minutos, casi el doble de lo que se tarda para ir de Zaragoza a Madrid (1 hora y 15 minutos) que está el doble de distancia y con muchos más accidentes geográficos y con 23 viajes de ida y vuelta diarios.

La posición estratégica de Zaragoza, entre Madrid-Barcelona, Bilbao-Valencia, ha contribuido a hacer de ellas uno de los nudos fundamentales en las comunicaciones ferroviarias a nivel nacional ya desde el siglo XIX. La ciudad llegó a tener cinco estaciones: Delicias, Arrabal, Madrid-ZaragozaAlicante (llamada del Santo Sepulcro) Utrillas y Cariñena. ${ }^{23} \mathrm{Su}$ construcción supuso modificaciones en el uso del espacio periurbano, en la vida y en la morfología de la ciudad, ya que éstas atraen en su entorno más próximo el asentamiento de población y la consiguiente localización de servicios complementarios. Son aun en la actualidad un elemento urbanístico y arquitectónico muy notable.

Hoy Zaragoza y su gran estación de Delicias cuenta claramente en el sistema ferroviario hispano, a medio camino entre MadridBarcelona y Navarra-Euskadi, pero ya no tiene ninguna esperanza de relación fructífera a través del ferrocarril con su territorio cercano. Así actualmente se sigue reivindicando por algunos sectores de la población aragonesa la mejora de las vías del tren Zaragoza- Canfranc como medio de conexión con Francia y El corredor Cantábrico-Mediterrraneo, pasando por Teruel.

El Atlas cartográfico de Zaragoza. Es una crónica de su historia-urbana que nos permitirá seguir las relaciones de la ciudad con su río, pero también con el tren, el canal y el territorio. Seguramente la mera visión y enumeración de sus puentes sea la forma más simple de evidenciar esta relación. Contra más puentes más intenso es el vínculo entre las riberas y son mejores el río y la ciudad.

Zaragoza posee, dada su condición estratégica y de gran ciudad, una buena colección de cartografias, extendida en el tiempo y en el espacio. Colección que se intensifica con motivo de los sitios de los franceses y con los planos de las reformas y ensanche del S. XIX y principios del XX. El Archivo Municipal de Zaragoza cuenta con un amplio catálogo cartográfico-histórico que nos ha permitido conocer la evolución y transformación experimentada por la ciudad.

Nos hemos fijado sobre todo en las cartografías que tienen que ver con el agua, sus propiedades y beneficios: las del Canal Imperial, las referentes al río Huerva y especialmente, las que tienen que ver con el Ebro mismo y sus puentes. Hemos puesto también especial atención en las que narran la posición de las primeras Estaciones Ferroviarias y los distintos $\mathrm{y}$ abundantes trazados del ferrocarril en la ciudad.

El Ebro para Zaragoza, durante siglos, ha sido un muro, una frontera, un límite insalvable para la ciudad. El puente de piedra es de 1441 y hasta finales del siglo XIX no hay otro, hasta que se construyen los puentes del ferrocarril y el de hierro. En el siglo XX tan solo se proponen tres puentes nuevos: el de Santiago (1967), el que conecta con la A2 (1978) y el de 
la Unión (1988). El gran salto se da a principios del 2000 con la construcción de 5 puentes, dos de ellos relacionados con los cinturones (tercer y cuarto cinturón). El resto corresponden a las infraestructuras de la Expo 2008 "precisamente situada en el meandro más anegable del Ebro": Pasarela del voluntariado, Puente del tercer Milenio y el Pabellón Puente. ${ }^{24} 25$

Entendemos que las cartografías, ordenadas cronológicamente, explican bien como la ciudad se ha ido acercando al río y finalmente ha conseguido extenderse por las dos riberas casi equilibradamente. La abundancia de sus puentes y la medida de su tejido confirman que en este larguísimo cortejo entre ciudad y río en que uno imperturbable: el río, y otra coqueta: la ciudad, se ha producido ininterrumpidamente hasta situar al río, casi en su centro de gravedad urbano.

\section{Conclusiones}

Es éste un trabajo incipiente, como desde el principio hemos anunciado, pero los abundantes e intencionados trabajos del curso y las reflexiones paralelas a esta comunicación, nos permitirá presentar algunas primeras conclusiones. La primera, la implícita dependencia de los dos actores del relato: Zaragoza y el Ebro.

Quizá sentados en los confortables sillones de la Confederación Hidrográfica del Ebro, delante del Panel de Control, con toda la Cuenca del Ebro monitorizada, desde la gran sala del Paseo Sagasta de Zaragoza podríamos pensar que la ciudad puede tomar todas las decisiones respecto al río. Los riesgos de las fluctuaciones del agua son ahora conocidos de antemano y los desembalses, decididos en relación a la posible lluvia, el deshielo o las necesidades de riego. Pero esa es una única y mínima forma de gobierno de la ciudad y su tecnología sobre el río. Él, el rio, entendido como una ecuación entre forma territorial y clima conserva toda su autoridad y autonomía respecto a su curso y respecto a la ciudad.

Menos evidente y más profunda es la acción del hombre sobre la propia agua que usa y abusa, embalsándola y regando - restándola del cauce - o produciendo energía que exportará a ciudades lejanas o produciendo alimento que enriquecen territorios. Pero el uso de sus abonos, herbicidas y fertilizantes contaminan indelebles sus aguas.

Zaragoza siempre y en cada momento, ha usado el rio de la manera más conveniente para ella. En el pasado fue defensa y vado: situada en un ligerísimo alto no inundable, la ciudad usó el Ebro y sus afluentes como límite defensivo. Incluso sus sotos, terrenos inundables, tenían ese fin. El Puente de Piedra, único en el centro del valle, es paso obligado y conforma la relación simbiótica entre la ciudad y él rio. El agua de los canales - El Imperial - llega a la ciudad en el S. XVIII y las tierras irrigadas permitirán agriculturas extensivas e intensivas. Gracias al azúcar de remolacha se produce: el crecimiento urbano, la vivienda obrera masiva y la consiguiente acumulación de capitales que permitirá convertir a Zaragoza en una gran urbe moderna.

La relación con el río y el agua ha cambiado. La ciudad extendida por las dos riberas, ha usado, por primera vez, sus aguas como argumento urbano, tendiendo sobre él nuevos puentes y pasarelas -y ya son 11- aunque algunos están especialmente infravalorados ${ }^{26}$. El Gallego es aún un límite, y a la vez una corriente no canalizada y poco contaminada, que quizá podría sustituir su vocación industrial por la de un corredor natural de la ciudad al Pirineo.

¿Tiene deudas la ciudad con su río? Claro!, y con sus territorios, de los que ha deseado y conseguido no solo agua sino a sus propios recursos y habitantes. Quizá sea la ambición desmedida de la ciudad para acumular, sobre su cuerpo, población y territorio ${ }^{27} \mathrm{El}$ territorio municipal de Zaragoza tiene 973,8 Km2 frente a los 102,0 Km2 de Barcelona, los 134,6 km2 de Valencia, los 140,8 de Sevilla o los 604,3 km2 de Madrid -a la fuerza- todos ellos núcleos de menor extensión. Este hecho es seguramente el que ha impedido el nacimiento de una periferia urbana con ciudades, primero en lucha con la ciudad central, pero constituyendo a la larga su metrópoli.

Quizá Zaragoza deberá ocuparse amorosamente de su propio entorno y territorio y devolverle agua, actividad $y$ 
hombres buscando un nuevo equilibrio. El Ebro imperturbable, con sus ciclos naturales y sus sitios culturales, podría contribuir a ello y sobre todo en su encuentro con la ciudad considerándose su mejor activo $\mathrm{y}$ aliado. La ciudad debe ser consciente de ello y los territorios fertilizados y los cascarones antes vacíos y revitalizados -ojalá- ahora le estarán agradecidos. Pero no es cuestión solo de cuentas $\mathrm{y}$ de equilibrios, sino de generosidad y de hospitalidad. La habitación del antiguo núcleo de Daroca, Calaceit o Albalate, abandonada en su día, ocupan aun su sitio y espera la vuelta de su usuario o propietario -o de uno nuevo- ser temporal o definitivamente habitada.

\section{Agradecimientos}

A la Confederación Hidrográfica del Ebro, especialmente a Miguel Ángel García y Adolfo
Álvarez, A Silvia Gonzales, Miguel Virgili, Sergio Vizarro, Josep María Solé, Arnau Tiñena,Stefano Cortellano, Pablo Roel, Daniel Marcelo y a todos los estudiantes de IV curso de proyectos y urbanismo ETSA/URV de Reus

\section{Notas}

1 Ciudad episcopal y puerta y puente sobre el Ebro fue la segunda ciudad más importante de las riberas del rio siempre después de Zaragoza. Hoy perdida su condición estratégica y parte de su territorio es una ciudad frustrada.

2 Es también una ciudad episcopal y con puente de piedra sobre el rio. Fue contrapunto de Pamplona y su fortuna se debe a la construcción de los canales que fertilizaron con las aguas del rio ambas riberas.

3 Ver Sòla-Morales, Manuel de. 1977. "las formas de crecimiento urbano". Universidad
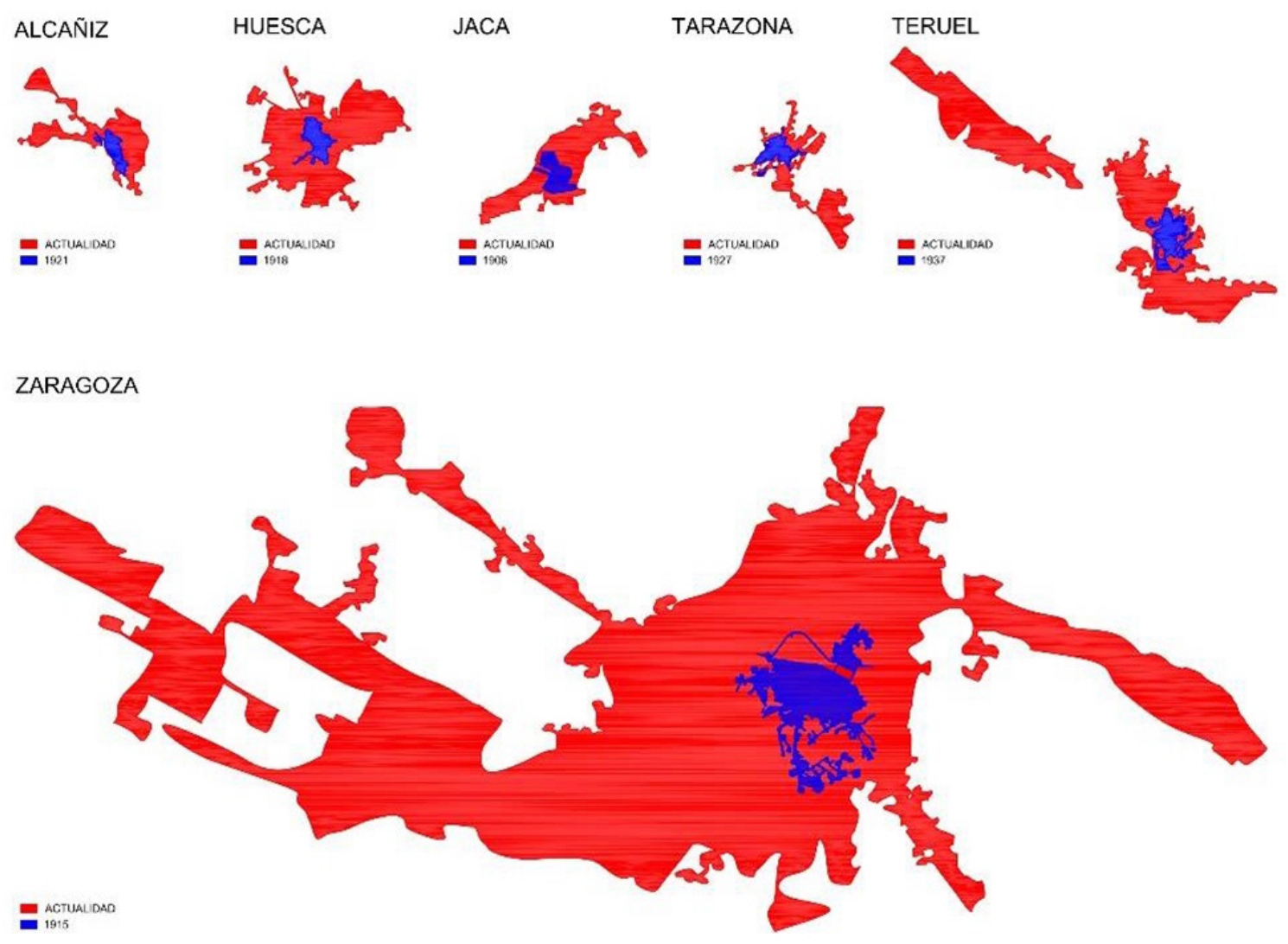

Fig. 1. Zaragoza-Huesca Teruel 

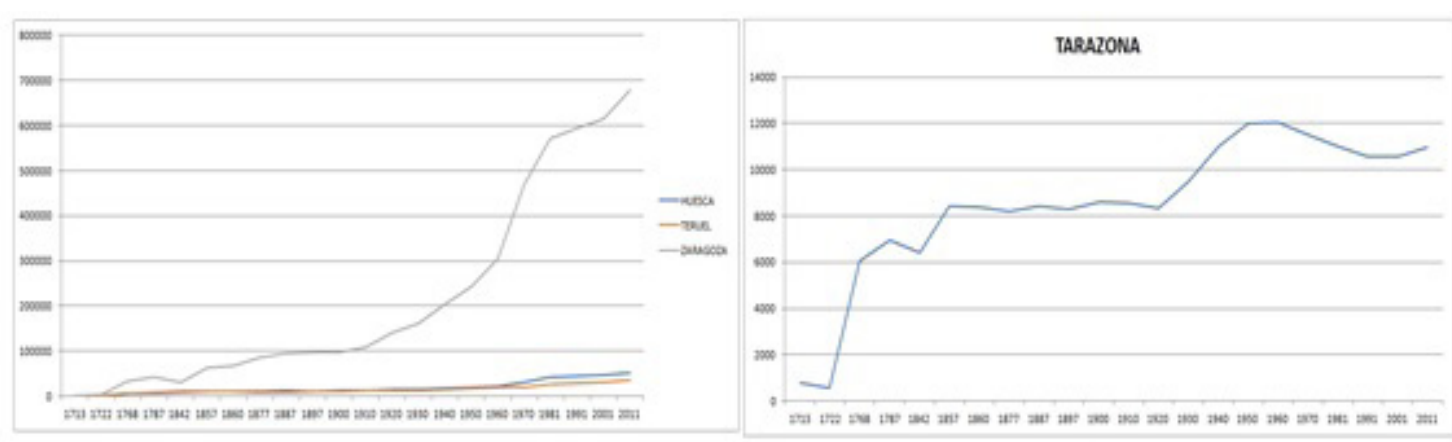

Figura 1. Zaragoza-Huesca Teruel
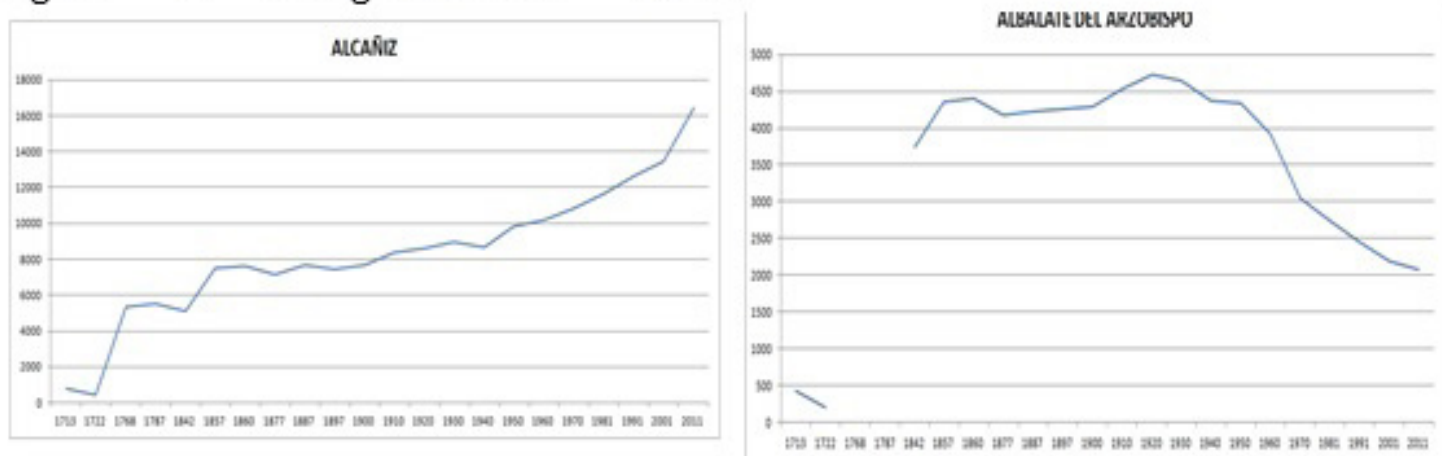

Figura 2 Tarazona

Figura3 AlcañizFigura

4 Albalate del Arzobispo
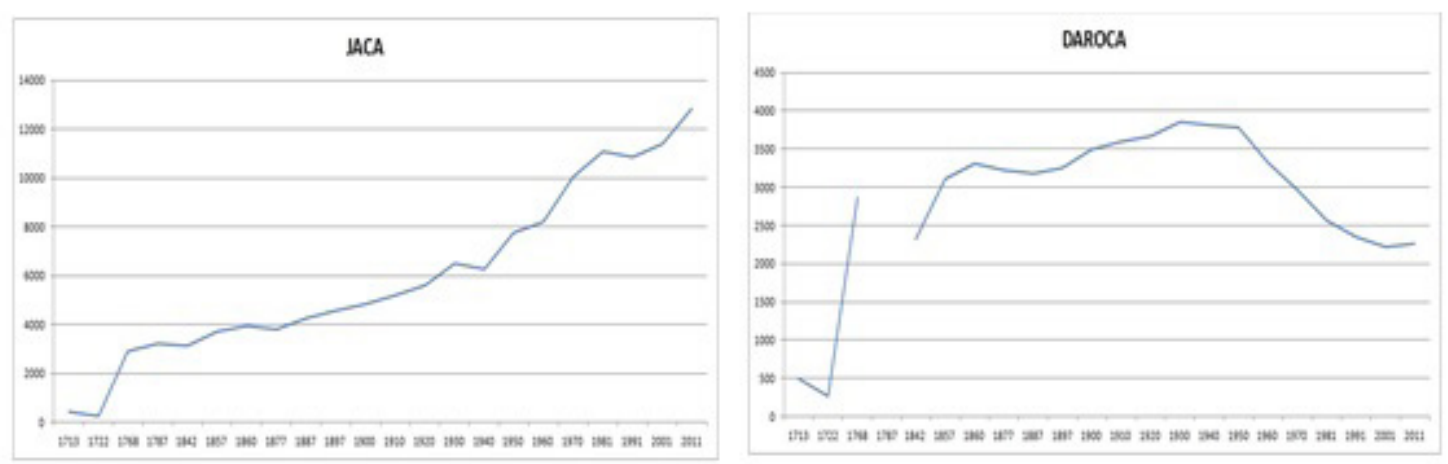

Figura 5 Jaca

Figura 6 Daroca

Graficas elaboradas por Solans, Burgui, Sarda (C.R.U.C.) 


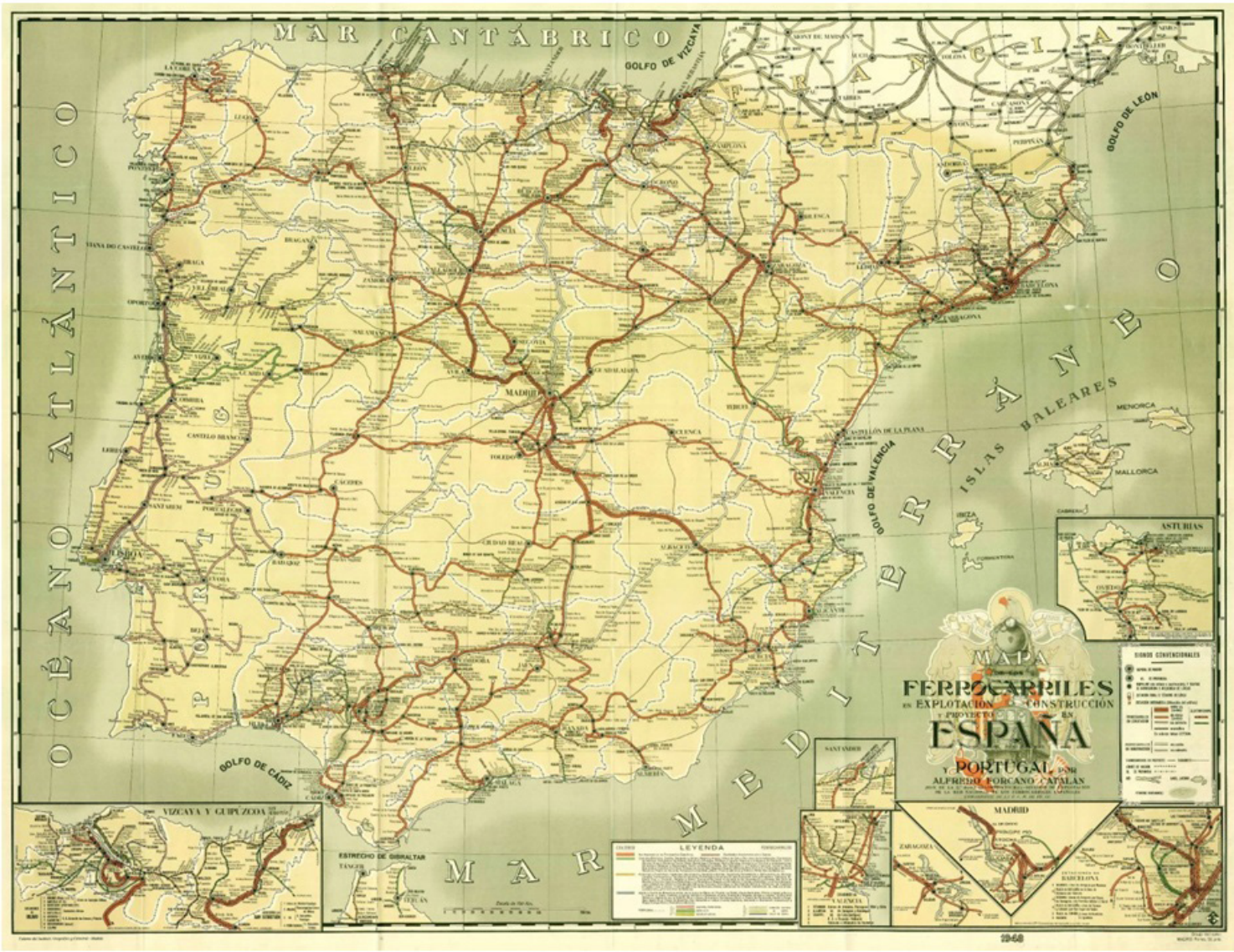

Fig. 7. Explotación, construcción y proyecto de los ferrocarriles de España y Portugal de Alfredo Forcano Catalán de 1949

Politècnica de Catalunya. Barcelona

4 Fue territorio extremo del municipio de Tortosa, y poco a poco un Delta habitado. Hoy los núcleos de Jesús i Maria y La Cava constituyen un único municipio: Deltebre.

5 Es una excepcional confluencia del Ebro con sus dos afluentes principales: el Segre y el Cinca. Es un castillo estratégico y un paisaje soberbio, pero también una presa hidroeléctrica y un pueblo antiguo inundado y reconstruido de nuevo en las proximidades. La fuerza de su pasado minero: Minas de Lignito -hoy completamente cerradas- construyen una historia tan trágica como excepcional.

6 Es la puerta del último escollo. Paso obligado del Ebro entre las montañas del sistema pre-litoral Catalán. El castillo templario certifica su condición estratégica.

7 Es un municipio navarro de la merindad de Tudela, recostado en las Bardenas Reales. Nos interesó por la posición y excepcionalidad de sus viviendas troglodíticas

8 Ver Magris, Claudio. 1986. "Danubio". Garzanti Editore. Milano

9 Ver Moncada, Jesús. 1988. "Camino de sirga". Ed. Anagrama. Barcelona

10 Es un poblado Ibérico poco inusual por su posición sobre el Ebro y la inhabitual planicie sobre el que está asentado. Es un extraordinario balcón sobre el río.

11 Ascó es un municipio antiguo y rico en monumentos: árabes, renacentistas, barrocos, pero hoy solo es reconocido por la presencia de su Central Nuclear, la única que resiste en el rio

12 Son los principales canales construidos a partir del siglo XIII transforman en tierras fértiles los antiguos eriales, su presencia tiene que ver con los nuevos paisajes agrícolas productivos y geométridos, pero también con la fortuna de las ciudades: Calahorra, Tudela, Zaragoza...

13 Las Bardenas Reales de Navarra, son 


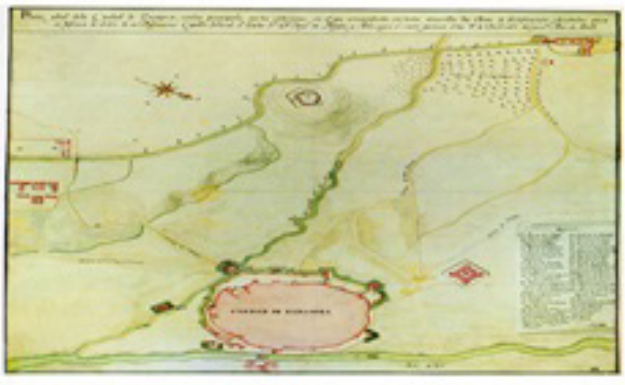

Figura 9. Plano ideal de la ciudad

de Zaragoza por Morata, año 1809

(Servicio Geográfico del Ejército núm. 115)

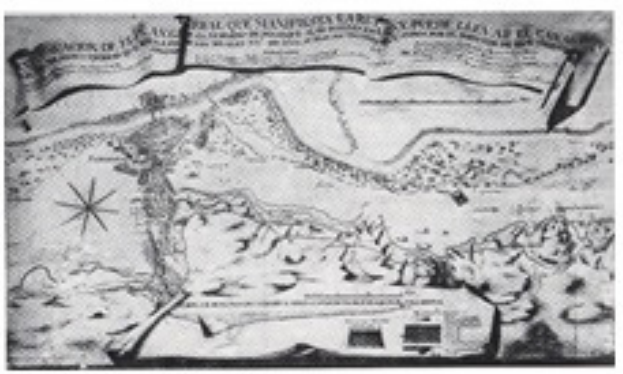

Figura 11 Plan General del Canal Imperial de Aragón por Joaquín de Villanova, año 1778

[Servicio Geográfico del Ejército núm. 139)

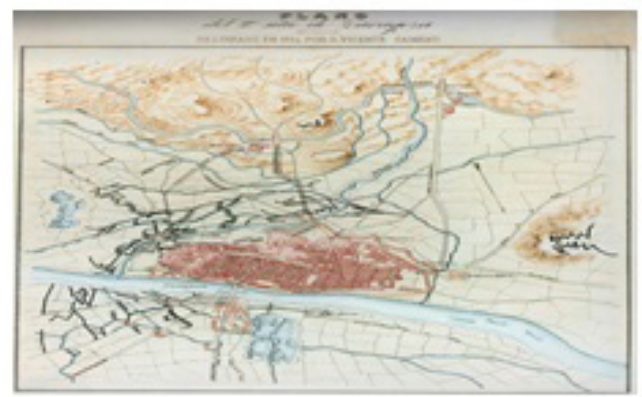

Figura 13. Plano del Segundo Sitio de Zaragoza por Vicente Gambád, año 1814

(servicio Geográfico del Ejército núm. 118)

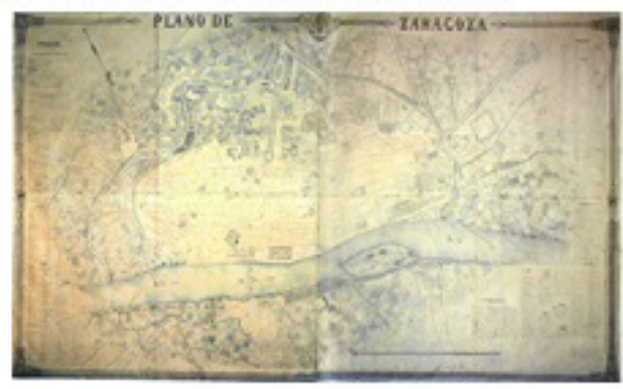

Figura 15. Plano de Zaragoza por J. Yarza y

」. Girroza, año 1853 (Colección particular)

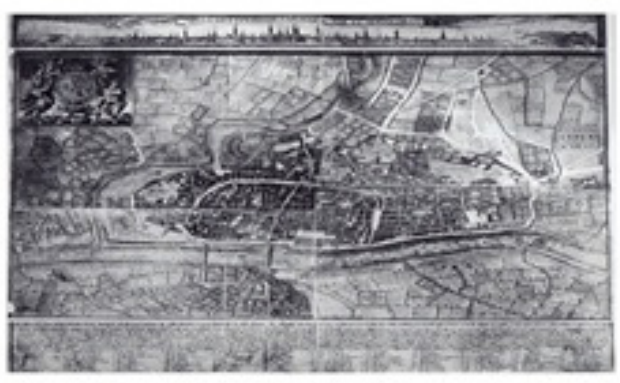

Figura 10. Vista de Zaragoza por el septentrión y plano, de Carlos Casanova, año 1734

_Servicio Geográfico del Ejército núm. 111)

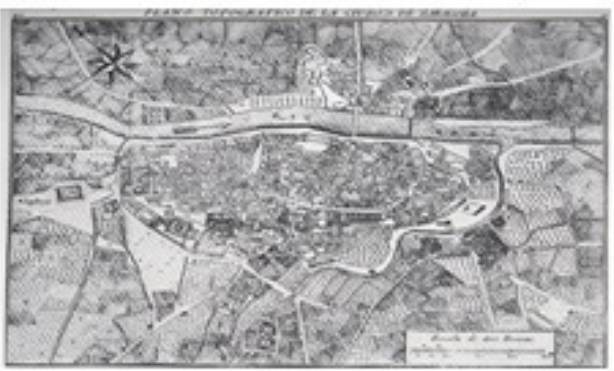

Figura 12. Plano Topográfico de la ciudad de Zaragoza, año 1778 (Colección particular)

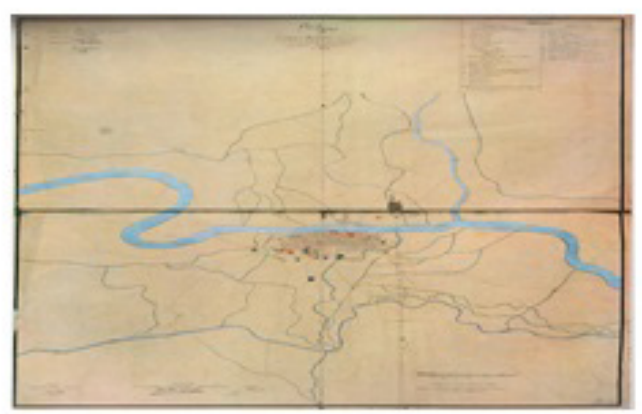

Figura 14. Plano de Zaragoza y sus cercanías, año 1847 (Servicio Histórico del Ejército núm. 2037)

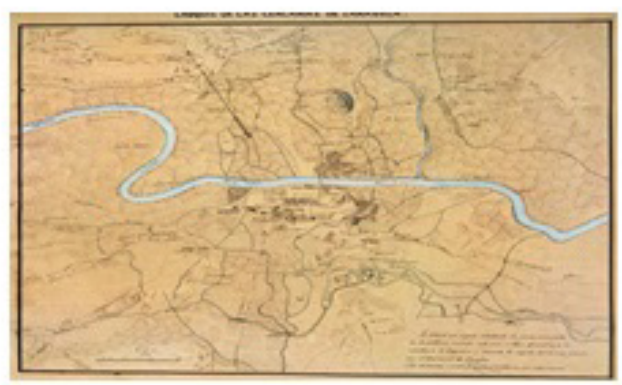

Figura 16. Croquis de las cercanías de Zaragoza (Servicio Histórico del Ejército núm. 2037) 


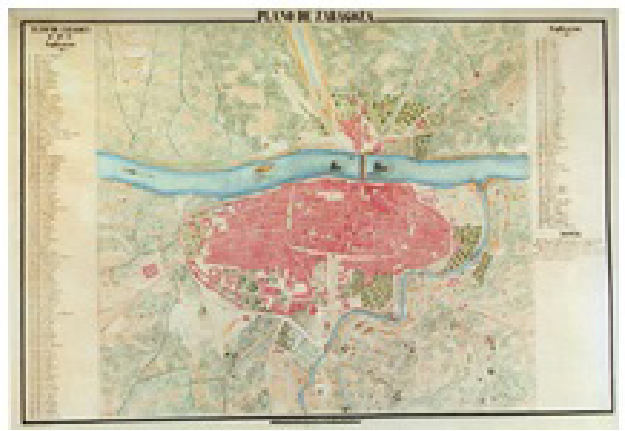

Figura 17. Plano de Zaragoza, 69 Dto. Por el cuerpo de E.M., año 1865(Servicio Geográfico del Ejército núm. 123)

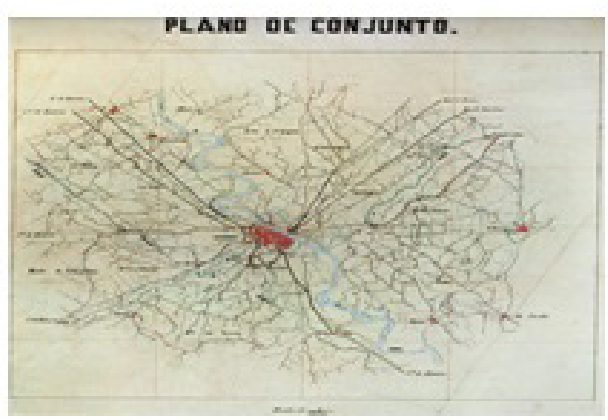

Figura 19. Plano de Conjunto, por el cuerpo de E.M, año 1869. (Servicio Geográfico del Ejército núm. 130)

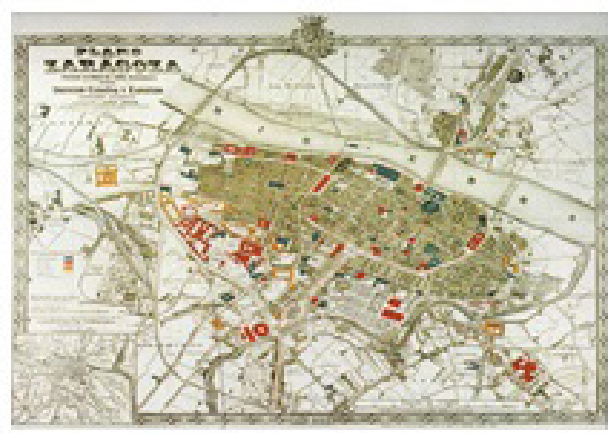

Figura 21. Plano de Zaragoza, por Dionisio Carsañale año 1908. (Colección particular)

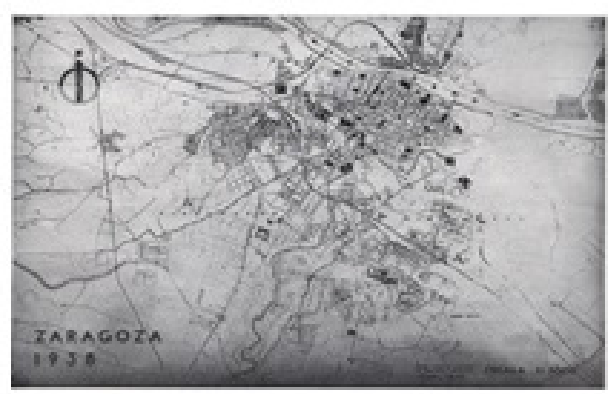

Figura 23. Plano de Zaragoza por Borobio y Beltrán. año 1938. (Ayuntamiento Zaragoza)

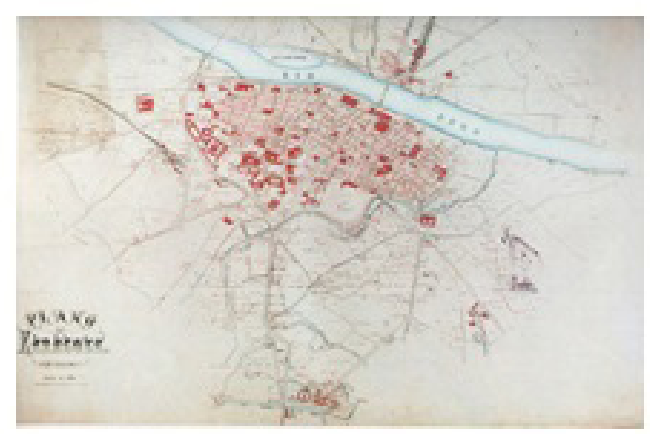

Figura 18 Plano de Zaragoza, Por el cuerpo de E.M año 1869 (Servicio Geográfico del Ejército núm. 1:

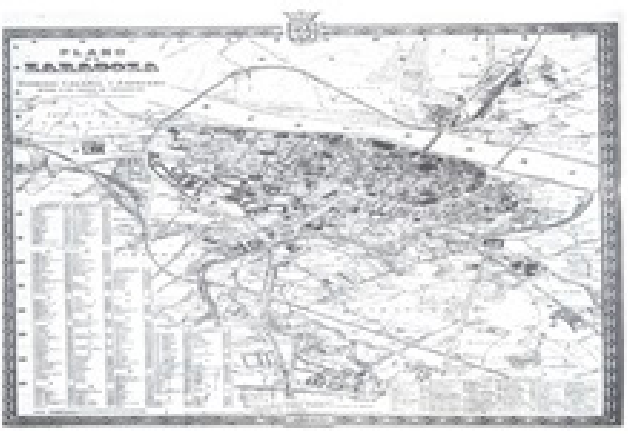

Figura 20. Plano de Zaragoza, por Dionisio Casañal año 1899. (Ayuntamiento Zaragoza)

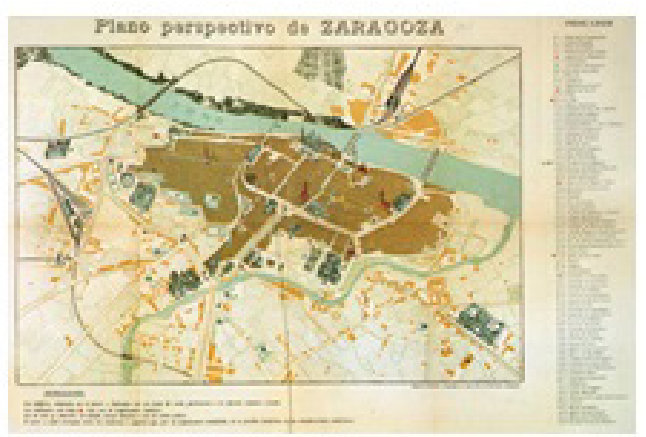

Figura 22. Plano perspectivo de Zaragoza

J. Galiax dibujó, año 1908. (Colección particular)

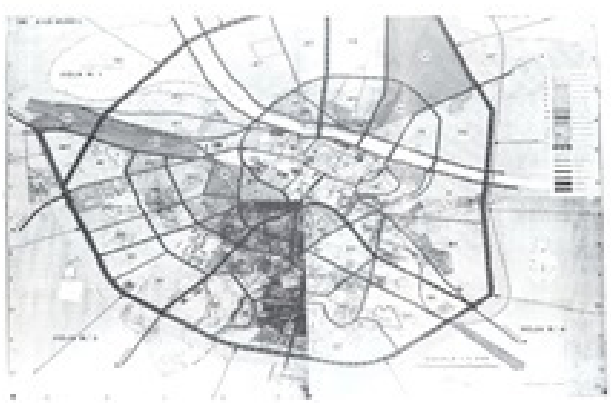

Figura 24. Anteproyecto de ordenación general, por Yarza, año 1943. (Ayuntamiento Zaragoza) 
Evolución histórica de la población desde 1900. Comarcas.

Reconstruceión según los términos municipales vigentes en el af́o 2001.

Unidad: personas.

Fuente: Censos de Población de 1900, 1910, 1920, 1930, 1940, 1950, 1960, 1970, 1981, 1991, 2001 y 2011

Poblaciones reconstruidas según la estructura de los términos municipales en 2011. IAEST

Metodologia

\begin{tabular}{|c|c|c|c|c|c|c|c|c|c|c|c|c|c|}
\hline \multirow[b]{2}{*}{$\begin{array}{l}\text { Comarca } \\
\text { código }\end{array}$} & \multirow[b]{2}{*}{ Comarca nombre } & \multicolumn{12}{|c|}{ Población } \\
\hline & & 1900 & 1910 & 1920 & 1930 & 1940 & 1950 & 1960 & 1970 & 1981 & 1991 & 2001 & 2011 \\
\hline \multicolumn{2}{|l|}{ Aragón } & 928.117 & 980.393 & 1.028 .255 & 1.051 .604 & 1.067 .661 & 1.090 .343 & 1.098 .887 & 1.153.055 & 1.196 .952 & 1.188 .817 & 1.204 .215 & 1.344 .544 \\
\hline 01 & La Jacetania & 26.996 & 27.017 & 26.478 & 26.904 & 24.908 & 24.270 & 21.167 & 17.926 & 16.948 & 16.007 & 16.676 & 18.178 \\
\hline 02 & Alto Gállego & 13.500 & 13.813 & 13.604 & 13.650 & 12.589 & 12.424 & 13.411 & 12.687 & 12.501 & 12.297 & 12.176 & 14.558 \\
\hline 03 & Sobrarbe & 22.277 & 22.667 & 22.761 & 21.826 & 20.303 & 18.290 & 15.122 & 9.525 & 7.091 & 6.638 & 6.803 & 7.663 \\
\hline 04 & La Ribagorza & 34.095 & 34.339 & 33.415 & 31.595 & 29.667 & 25.694 & 23.201 & 16.665 & 12.953 & 11.915 & 11.792 & 13.037 \\
\hline 05 & Cinco Villas & 36.105 & 38.487 & 43.611 & 45.889 & 45.677 & 42.682 & 40.779 & 37.640 & 34.757 & 32.656 & 32.209 & 32.502 \\
\hline 06 & $\begin{array}{l}\text { Hoya de Huesca / } \\
\text { Plana de Uesca }\end{array}$ & 54.181 & 56.183 & 56.536 & 57.590 & 54.325 & 54.618 & 52.338 & 53.374 & 58.074 & 58.743 & 60.254 & 67.921 \\
\hline 07 & $\begin{array}{l}\text { Somontano de } \\
\text { Barbastro }\end{array}$ & 33.324 & 33.406 & 32.898 & 29.124 & 28.361 & 26.211 & 25.978 & 24.804 & 24.094 & 23.105 & 22.772 & 24.309 \\
\hline 08 & Cinca Medio & 16.350 & 17.736 & 17.578 & 17.354 & 17.438 & 17.338 & 20.102 & 23.989 & 22.947 & 22.265 & 22.132 & 24.031 \\
\hline 09 & $\begin{array}{l}\text { La Litera / La } \\
\text { Litera }\end{array}$ & 17.917 & 20.267 & 21.767 & 20.872 & 21.117 & 21.655 & 21.940 & 21.831 & 20.761 & 19.477 & 18.487 & 18.852 \\
\hline 10 & Los Monegros & 28.881 & 30.951 & 31.753 & 30.616 & 30.548 & 28.234 & 28.841 & 27.698 & 25.661 & 23.253 & 20.989 & 20.580 \\
\hline 11 & $\begin{array}{l}\text { Bajo Cinca / Baix } \\
\text { Cinca }\end{array}$ & 22.233 & 24.254 & 25.492 & 23.303 & 22.993 & 23.209 & 26.033 & 23.475 & 22.978 & 22.666 & 22.254 & 24.552 \\
\hline 12 & $\begin{array}{l}\text { Tarazona y el } \\
\text { Moncayo }\end{array}$ & 19.078 & 18.372 & 18.730 & 20.084 & 21.251 & 22.396 & 20.145 & 17.636 & 15.671 & 14.520 & 14.168 & 14.557 \\
\hline 13 & Campo de Borja & 22.880 & 21.447 & 22.043 & 21.428 & 21.241 & 21.272 & 19.616 & 17.254 & 15.580 & 14.632 & 14.181 & 15.166 \\
\hline 14 & Aranda & 12.482 & 12.967 & 13.281 & 13.859 & 13.105 & 12.490 & 10.780 & 8.984 & 8.438 & 8.248 & 7.967 & 7.402 \\
\hline 15 & $\begin{array}{l}\text { Ribera Alta del } \\
\text { Ebro }\end{array}$ & 16.964 & 19.011 & 22.222 & 24.628 & 25.769 & 25.629 & 26.437 & 24.551 & 21.728 & 21.622 & 22.286 & 27.761 \\
\hline 16 & Valdejalón & 26.396 & 29.173 & 30.995 & 30.801 & 31.209 & 30.702 & 28.960 & 25.268 & 22.715 & 21.354 & 22.865 & 29.671 \\
\hline 17 & D.C. Zaragoza & 118.012 & 131.838 & 163.029 & 185.659 & 228.571 & 268.770 & 330.140 & 496.531 & 600.740 & 626.447 & 656.746 & 752.937 \\
\hline 18 & $\begin{array}{l}\text { Ribera Baja del } \\
\text { Ebro }\end{array}$ & 15.281 & 15.974 & 15.787 & 15.120 & 14.156 & 14.894 & 14.642 & 12.594 & 10.817 & 10.104 & 9.211 & 9.366 \\
\hline 19 & $\begin{array}{l}\text { Bajo Aragón- } \\
\text { Caspe / Baix } \\
\text { Arago-Casp }\end{array}$ & 17.634 & 19.588 & 20.580 & 20.066 & 19.633 & 19.538 & 18.398 & 16.119 & 14.563 & 13.554 & 13.005 & 14.935 \\
\hline 20 & $\begin{array}{l}\text { Comunidad de } \\
\text { Calatayud }\end{array}$ & 68.998 & 70.740 & 74.364 & 80.262 & 83.848 & 81.981 & 71.999 & 56.851 & 47.355 & 41.954 & 39.144 & 40.934 \\
\hline 21 & $\begin{array}{l}\text { Campo de } \\
\text { Cariñena }\end{array}$ & 16.997 & 18.228 & 18.120 & 17.238 & 16.937 & 16.903 & 14.705 & 12.731 & 11.362 & 10.173 & 10.243 & 11.053 \\
\hline 22 & Campo de Belchite & 15.133 & 16.846 & 17.361 & 17.557 & 16.103 & 16.197 & 12.827 & 9.254 & 6.982 & 6.147 & 5.530 & 5.198 \\
\hline 23 & Bajo Martin & 15.605 & 16.979 & 17.490 & 17.502 & 16.362 & 15.992 & 14.185 & 11.292 & 9.665 & 8.484 & 7.329 & 6.932 \\
\hline 24 & Campo de Daroca & 20.409 & 21.181 & 21.779 & 22.479 & 21.952 & 21.677 & 17.568 & 11.807 & 8.779 & 7.360 & 6.467 & 6.203 \\
\hline 25 & Jiloca & 34.372 & 36.532 & 37.440 & 40.709 & 40.089 & 38.857 & 33.548 & 23.700 & 18.470 & 15.640 & 13.653 & 13.590 \\
\hline 26 & Cuencas Mineras & 22.525 & 24.444 & 23.530 & 23.197 & 21.349 & 22.813 & 22.222 & 16.255 & 14.109 & 11.226 & 9.485 & 8.883 \\
\hline 27 & $\begin{array}{l}\text { Andorra-Sierra de } \\
\text { Arcos }\end{array}$ & 12.756 & 14.226 & 13.881 & 12.963 & 12.736 & 14.096 & 16.918 & 12.242 & 12.428 & 12.606 & 11.034 & 11.209 \\
\hline 28 & Bajo Aragón & 32.459 & 34.396 & 33.795 & 33.259 & 31.451 & 30.401 & 28.383 & 25.837 & 25.597 & 26.195 & 26.516 & 30.037 \\
\hline 29 & $\begin{array}{l}\text { Comunidad de } \\
\text { Teruel }\end{array}$ & 40.244 & 44.032 & 45.367 & 49.102 & 47.450 & 51.188 & 49.979 & 42.867 & 42.345 & 42.243 & 42.767 & 47.068 \\
\hline 30 & Maestrazgo & 20.648 & 20.428 & 18.803 & 17.415 & 15.352 & 13.711 & 11.350 & 7.389 & 4.964 & 4.184 & 3.713 & 3.579 \\
\hline 31 & $\begin{array}{l}\text { Sierra de } \\
\text { Albarracin }\end{array}$ & 15.793 & 16.021 & 16.047 & 15.776 & 14.114 & 13.667 & 12.132 & 8.631 & 6.093 & 5.417 & 4.903 & 4.918 \\
\hline 32 & Gúdar-Javalambre & 34.069 & 34.809 & 33.292 & 30.816 & 26.937 & 24.264 & 19.827 & 13.173 & 9.139 & 8.082 & 7.742 & 8.278 \\
\hline 33 & $\begin{array}{l}\text { Matarrafa / } \\
\text { Matarranya }\end{array}$ & 23.523 & 24.041 & 24.417 & 22.961 & 20.120 & 18.280 & 15.214 & 12.475 & 10.647 & 9.603 & 8.716 & 8.684 \\
\hline
\end{tabular}

Fig. 25. Evolución histórica de la población de Aragón por comarcas desde 1900 a 2011. IAEST.

una meseta semidesértica, hoy parcialmente cultivada pero aun no habitada

14 Es el organismo dependiente del Ministerio de Fomento que gobierna con autoridad sobre todas las cuestiones hidráulicas de la Cuenca del Ebro

15 Ver Sales, Joan. Inserta Gloria. Ed. Aymà i Club Editor. Barcelona. 1956.

16 Ver Villaronga, Agusti. 2017. Inserta Gloria. Masa d'or i Tv de Catalunya. España

17 Ver Malraux, André. 1937 Espoir, sierra de Teruel. Paris, Gallimard, collection

18 Ver Malraux, André. 1939 Espoir, sierra de Teruel. Productora Corniglion-Molinier. Francia

19 Como se puede observar en la figura 1 la expansión del casco urbano de Zaragoza lo hace de manera desproporcionada frente al resto de ciudades aragonesas

20 Ver figura 8

21 Como en tantos lugares de Europa el ferrocarril tiene en el Valle del Ebro un 
doble sentido el de transporte de viajeros y el industrial vinculado al carbón y al hierro. Este último, sobre todo en Aragón, fracasó. Solo queda el transporte de viajeros, cada vez más rápido y con mayor eficacia, capacidad $\mathrm{y}$ productividad, pero solo entre las grandes ciudades

22 El puente de hierro sobre el Ebro, se inauguró en 1870. Su construcción se llevó a cabo para que el tren procedente de Navarra pudiera llegar hasta la estación del Arrabal. En 1970 los trenes dejaron de circular por el puente, $\mathrm{y}$ se acondiciono para el paso de coches y peatones

23 Las estaciones de Utrillas y Cariñena se construyeron respondiendo a intereses económicos locales. La de Utrillas para el transporte del carbón de las zonas mineras de Utrillas-Escatrón. Actualmente, esta estación es un centro comercial, quizá si se hubiera mantenido el servicio ferroviario podría ser una estación de cercanías. La de Cariñena estaba más relacionada con los productos agrícolas de la zona como el vino. El resto de las estaciones se plantearon dentro de las estrategias de las compañías ferroviarias, pensando más en las relaciones interregionales que en las locales.

24 Ver Monclús, Javier y Blasco, Jerónimo. 2007. "La Expo Zaragoza 2008 proyecto urbano estratégico". En Planes y proyectos para la ciudad 2000-2015, pag 103-112. Cámara de Comercio e Industria de Zaragoza. Zaragoza.

25 Monclús, Javier. Diaz, Carmen y Cal, Pablo de la. 2018. "Nuevas miradas y exploraciones urbanas, Zaragoza 1968-2018". COAA. Zaragoza

26 E incluso sin uso como el Pabellón Puente construido para la Expo 2008 y diseñado por Zaha Hadid.

27 Ver figura 20

\section{Referencias}

Adiego, Elvira. 2002 "Visión histórica y urbana de los ríos en Zaragoza" En Ríos y Ciudades: Aportaciones para la recuperación de los ríos y riberas de Zaragoza, pag 251271. Institución "Fernando el Católico" (C.S.I.C). Zaragoza.

Barrera, Mariano. 2007. "Confederación hidrográfica del Ebro (1926-2001) apuntes para una historia". Ed. Prames. Zaragoza.

Cal, Pablo de la. 2018. "las formas del agua en la regeneración urbana de Zaragoza. Recuperación de la red de riego como estrategia de la construcción de lugares", pag 77-86. doi: 10.26754/uz. 9788417358820. Zaragoza.

Cases, Pedro. 2008 "El Ebro viaje por el camino del agua". Ed. Península. Barcelona.

Faus, M ${ }^{\mathrm{a}}$ Carmen. 1978. "El ferrocarril y la evolución urbana de Zaragoza", pag 83-114 doi.org/10.26754/ojs.geoph/ geoph.197822791. Zaragoza.

Gaviria, Mario. 2002. "El agua y la ciudad" En Ríos y Ciudades: Aportaciones para la recuperación de los ríos y riberas de Zaragoza, pag 33-44. Institución "Fernando el Católico" (C.S.I.C). Zaragoza.

Gaviria, Mario, Grillo, Enrique. 1974. "Zaragoza contra Aragón" Libros de la frontera. Barcelona.

Germán, Luis.1989. "La transformación de la ciudad. Zaragoza en el siglo XX (19001936)" En Evolución Histórico-urbanística de la ciudad de Zaragoza Vol I, pag 459468. Ed. Colegio Oficial de Arquitectos de Aragón. Zaragoza.

Laborda, José. 2001. "Confederación hidrográfica del Ebro, Zaragoza 19331946. Regino y José Borobio Ojeda". Ed. Colegio Oficial de Arquitectos de Andalucía Oriental, Delegación de Almería. Barcelona.

Magris, Claudio. 1986. "Danubio". Garzanti Editore. Milano.

Malraux, André. 1937 Espoir, sierra de Teruel. Paris, Gallimard, collection.

Malraux, André. 1939 Espoir, sierra de Teruel. Francia. Productora Corniglion-Molinier

Martinez, Jesús. 2015. "Zaragoza: Arquitectura: Siglo XX”. Ed. Lulu. Carolina del Norte.

Moncada, Jesús. 1988. "Camino de sirga". Ed. Anagrama. Barcelona.

Monclús, Javier y Blasco, Jerónimo. 2007. "La Expo Zaragoza 2008 proyecto urbano estratégico". En Planes y proyectos para la ciudad 2000-2015, pag 103-112. Cámara de Comercio e Industria de Zaragoza. Zaragoza. Monclús, Javier. Diaz, Carmen y Cal, Pablo de la. 2018. "Nuevas miradas y exploraciones urbanas, Zaragoza 1968-2018”. COAA. Zaragoza. 
Pellicer, F. 2003. "Ciudades y ríos de la cuenca del Ebro". Ed. Heraldo de Aragón. Zaragoza.

Sales, Joan. Inserta Gloria. Ed. Aymà i Club Editor. Barcelona. 1956.

Sòla-Morales, Manuel de. 1977. "las formas de crecimiento urbano". Universidad Politècnica de Catalunya. Barcelona.

Villaronga, Agusti. 2017. Inserta Gloria. Masa d'or i Tv de Catalunya. España

Yeste, Isabel. 2009. "Del centenario de los sitios a la exposición internacional de 2008" En La ciudad de Zaragoza de 1908 a 2008", pag 11-62. Institución Fernando el Católico (C.S.I.C). Zaragoza. 